\title{
Cytogenetics of Primary Skin Tumors
}

\author{
Melanie A Carless and Lyn R Griffiths
}

Skin tumors can arise as a result of cumulative genetic abnormalities including chromosomal aberrations that can be described as either morphological (structural rearrangements) or molecular (copy number variations). Cytogenetic techniques have been used to examine both large and small chromosomal aberrations, and include karyotyping, comparative genomic hybridization, and fluorescence in situ hybridization. This chapter describes the recurrent aberrations associated with skin tumors, such as benign melanocytic nevi, melanoma, basal cell carcinoma, squamous cell carcinoma, actinic (solar) keratosis, Bowen’s disease, keratoacanthoma, Merkel cell carcinoma, dermatofibrosarcoma protuberans and cutaneous lymphomas as detected by cytogenetic methodologies. A significant number of genomic aberrations are shared across different subtypes of skin tumors, including structural and numerical alterations of chromosome 1 , $-3 p,+3 q,+6,+7,+8 q,-9 p,+9 q,-10,-17 p,+17 q$ and +20 . Aberrations specific to certain skin cancers have also been detected and include loss of $18 q$ in squamous cell carcinoma but not its precursor, actinic keratosis; loss of 9q22 in sporadic basal cell carcinoma; and translocation involving $17 q 22$ and $22 q 13$ in dermatofibrosarcoma protuberans. These regions contain a number of potential candidate genes that are involved in aspects of cell signaling, proliferation, differentiation and apoptosis. Cytogenetic methodologies continue to evolve with the advent of array-based comparative genomic hybridization, copy number variation microarrays and next-generation sequencing. It is envisioned that cytogenetic analysis will continue to be employed for identification and further 
exploration of novel chromosomal regions and associated genes that drive skin tumorigenesis.

\section{Introduction}

Since the late 1950's, cytogenetic techniques have been employed to investigate chromosomal aberrations contributing to human disease, including those associated with solid and hematological tumors (1). Historically, it has been considered the gold standard for prenatal diagnosis of chromosomal abnormalities, as well as for the diagnosis and prognostication of a number of human cancers, including chronic myeloid leukemia (CML) (2), myelodysplastic syndrome (3) and Ewing sarcoma (4). Cytogenetic analyses of pre-malignant and malignant skin lesions have identified a number of aberrant regions that appear to contribute to disease development and progression.

\section{Technologies}

Some of the earliest cytogenetic studies investigated large chromosomal rearrangements that were easily detectable by microscopy using chromosomal banding patterns (i.e.; G-banding). The identification of the Philadelphia chromosome in 1960 (5), later defined as a translocation between chromosomes 9 and 22 [t(9;22)] (6), was the first chromosomal abnormality found to be consistently associated with a specific malignancy (CML). Since then, many translocations, duplications and inversions have been identified in various malignancies utilizing banding techniques. While karyotypic testing of hematological malignancies is extremely successful in determining chromosomal abnormalities, the analysis of solid tumors has been much more problematic, due to the 
necessity for short- or long-term cell culture in the latter, including skin tumors. Specifically, growth of melanoma and non-melanoma skin cancer (NMSC) cell cultures have identified preferential growth of contaminating stromal fibroblasts in NMSC (7) and subclone selection in melanoma (8). Caution is therefore warranted when interpreting cytogenetic abnormalities detected in samples derived from cultured skin tumors.

Comparative genomic hybridization (CGH) is a fluorescent-based technique that alleviates some of the problems associated with cell culturing, as DNA derived directly from a tumor sample can be used as a template to globally screen for gross (>20mb) copy number aberrations (9). Whole-genome amplified and differentially labeled tumor and reference DNA are co-hybridized to normal metaphase spreads, such that imbalances are detected by changes in fluorescence values of the tumor DNA relative to the reference DNA $(9,10)$. The main drawback of CGH analysis is that it detects only relatively large numerical or unbalanced alterations. However it does not require the interpretation of complex tumor karyotypes or prior knowledge of aberrations for probe design, and can therefore easily detect previously unknown DNA copy number variation (CNV) $(9,10)$.

Fluorescence in situ hybridization (FISH), detects copy number changes, translocations and inversions that might otherwise be difficult to detect using standard karyotypic analysis (10-12). The resolution of FISH is much higher than that of CGH (10-12). FISH is applicable to both interphase and metaphase chromosomal analysis, but requires the design of sequence-specific probes that can be large (up to a whole chromosome) or small (as low as 1-200kb), and therefore prior knowledge of the aberrant region is required (10-12).

Loss of heterozygosity (LOH) analysis, although not a cytogenetic technique per 
se, is often used to confirm and better resolve regions associated with loss of genetic material detected by other methodologies. Highly polymorphic markers or specific genes are used to determine the presence of genetic material in both normal and tumor DNA from an individual, with allelic loss in the tumor tissue correlating with a deleted chromosomal region (13).

More recently, detection of copy number and structural aberrations has reached a new standard with the introduction of array-CGH (14), CNV-microarrays (15) and whole-genome sequencing (16). In conjunction with other molecular techniques (outlined in Chapter 3), cytogenetic analysis is a powerful means to identify genetic factors that drive tumorigenesis and has been employed in the study of skin tumors. The determination of numerical and/or structural chromosomal aberrations, accompanied by further refinement of these regions, will facilitate the identification of putative oncogenes and tumor suppressor genes associated with cancer development and progression.

In addition, a number of online databases exist that summarize cytogenetic aberrations in human cancers [for a review see (17)], including the Mitelman Database of Chromosome Aberrations in Cancer (18) and the Atlas of Genetics and Cytogenetics in Oncology and Hematology (19).

\section{Primary Skin Cancers}

Cancer of the skin describes the uncontrolled growth of cutaneous cells, arising from an accumulation of inherited and/or sporadic genetic abnormalities, and with the potential for metastatic spread to other organ systems. Classified into two broad categories, melanoma and NMSC, skin cancers are some of the most common human tumors in 
many countries and their incidence is rapidly increasing. NMSC include basal cell carcinoma (BCC), squamous cell carcinoma (SCC), actinic keratosis (AK), SCC in situ, and keratoacanthoma (KA) (20). Other primary skin cancers include merkel cell carcinoma (MCC), dermatofibrosarcoma protuberans (DFSP) and cutaneous lymphomas $(20,21)$. Extensive cytogenetic analysis of many of these tumors has been conducted and reveals a number of recurrent aberrations that are likely to be associated with the development or progression of skin cancer.

\section{Melanocytic Skin Tumors}

Cutaneous melanoma is the most deadly form of skin cancer (22). In the United States, it is estimated that, in $2009,68,720$ individuals will be diagnosed with melanoma ( 22.6 per 100,000 individuals) and that 8,650 individuals will die from this disease ( $\sim 2.8$ per 100,000 individuals) (22).

Summarized information from the Atlas of Genetics and Cytogenetics in Oncology and Hematology indicates that the most common karyotypic aberrations associated with melanoma are deletions and translocations involving chromosomes 1 and 6q; gain of 6p, which may play a role in cancer progression; and gain of chromosome 7, which is associated with late stages of the disease (19). Höglund et al. (23) authored a comprehensive review of genetic changes in melanoma using previously published data obtained from the Mitelman Database of Chromosome Aberrations in Cancer (18). They noted that most common aberrations detected were -10 (59\%), -6q10-q27 (42\%), $-9 p 10-p 24,-21(37 \%),+7,-16$ (36\%), -14, +1q24-q44, -4, -15 (33\%), -5 (32\%), -1p10-p36, -11q23-q25 (28\%), -12q13-q24, +20 (27\%), -17p, +18 (26\%), -8p10-p23, 
+8q10-q24 (25\%), -3 (24\%), -22, -X (23\%), +6p21-p25, -18 (22\%), +3 (18\%), -19 (17\%),

+9q22-q34 (15\%), +19 (14\%), +13, +17q10-q25 (12\%), +2, +15, +21 and +22 (11\%)

(23). CGH analysis has also identified a number of chromosomal regions with recurrent aberrations in melanoma. Bastian et al. (24) studied 132 melanomas and identified recurrent gain of 6p (37\%), 1q (33\%), 7p, 7q (32\%), 8q (25\%), 17q (24\%) and 20q (22\%), and recurrent loss of 9p (64\%), 9q, 10q (36\%), 10p (30\%), 6q (26\%) and 11q (21\%). Genomic imbalance in melanoma is common, with chromosomal gain and loss being reported on all chromosomes (18). In addition to these aberrations, which result in an imbalance of genetic material, balanced translocations involving regions on 1q, 6q, 14q and $19 \mathrm{p}$ have also been identified, although in a smaller percentage of cases (18).

It has been hypothesized that there are key cytogenetic events that drive development and progression of human melanocytic tumors. Karyotypic investigations of benign melanocytic nevi (BMN) have identified translocations as a principal cytogenetic event. Richmond et al. (25) identified single occurrences of reciprocal translocations involving $\mathrm{t}(6 ; 15), \mathrm{t}(10 ; 15), \mathrm{t}(15 ; 20)$ and $\mathrm{t}(4 ; 5)$ in an investigation of eight BMN. In addition, three BMN from a single patient with a family history of melanoma were all found to have simple translocations; one of which included a $6 q 13$ breakpoint (26), a region also implicated (deleted) in melanoma (18). Bastian et al. (24) performed CGH analysis on $54 \mathrm{BMN}$ and found that only 13\% exhibited CNV. Of the seven BMN that exhibited aberrations, six of these showed a gain of 11p (all Spitz nevi) (24). This aberration was not found in any of the melanomas studied (24). Early chromosomal instability detected in BMN could define lesions that have a higher potential for oncogenic transformation; although specific chromosomal aberrations may not 
necessarily be consistent during such transformation. Recurrent alterations, predominantly deletions, have been identified on chromosome 9 in both dysplastic melanocytic nevi and metastatic melanoma lesions $(19,27)$, suggesting that this may be a primary event in melanocytic transformation. Höglund et al. (23) suggested two major karyotypic pathways as early cytogenetic changes in melanoma: (i) one involving $+6 \mathrm{p}$, $-6 q$ and possibly -16 and (ii) a second involving -3 and either $+8 q$ or $-8 p$. A CGH study investigating 16 primary melanomas and 12 metastatic tumors identified gains of 5p, 5q21-q23, 10p and 18q, as well as losses of 2p21-pter, 11q13-q23, 12q24.1-qter, 19q13.1-qter and 22qter in metastatic lesions but not in primary lesions (28). Also, losses involving 9p and 17 occurred at a higher frequency in metastatic tumors (28). In cases where the primary and metastatic lesions $(n=4)$ were excised from the same patient, metastases were associated with the acquisition of additional aberrations; although none of these were determined to be recurrent (28).

FISH and $\mathrm{LOH}$ analyses have been used to confirm and further investigate aberrant genomic regions in melanoma, previously identified by karyotypic and CGH analysis. Studies have identified: (i) extra copies (89\%) and translocations (25\%) of chromosome 20 (whole chromosome painting) (29); (ii) extra copies of c-myc (8q24.21) in nodular (61\%) and superficial spreading (27\%) melanomas (30); (iii) copy number gains of 7 (40.9\%), 6, 17 (27\%), 9 and 10 (23\%) and monosomies of 10 (55\%), 9 (37\%), 6 (27\%), 17 (23\%), 1 and 7 (18\%) (31); and (iv) polysomy of chromosome 7 (67\%) that is associated with amplification of EGFR (epidermal growth factor receptor), as well as common alterations of $+6,+8,-9$ and -10 (32). A higher frequency of $\mathrm{LOH}$ on $1 \mathrm{p}$ and $9 \mathrm{p}$ is found in melanoma ( $29 \%$ and $50 \%$ at the most frequently lost loci) compared to 
dysplastic nevi (12\% and 27\% at corresponding loci) and BMN (no LOH) (33). Uribe et al. (34) found loss of 9p21, 17q21, 6q23, and 5q35 more frequently in melanoma (68\%), compared to atypical nevi (57\%) and BMN (27\%). In another study of 13 cases of early-stage melanoma, LOH was detected for at least one locus at 9p22 (31\%), 10q11 (31\%) and 1p36 (15\%) (35). Udart et al. (36) determined that metastatic melanomas have higher rates of +7 (25\%) compared to primary lesions (8\%). However, a higher prevalence of additional copies of CCND1 (11q13) is found in primary (47\%) versus metastatic (35\%) lesions (37). Although there is not a clear model to describe the influence of $\mathrm{CNV}$ on the development and progression of melanoma, studies to date suggest that a number of chromosomal regions may play a role in the early formation of cancerous cells (9p) as well as a metastatic phenotype $(7,11 q)$. Figure $4-1$ summarizes using Circos (38), the recurrent aberrations detected in melanoma samples by karyotypic, CGH, FISH and LOH techniques.

\section{Keratinocytic Skin Tumors}

The American Cancer Society estimates that more than one million cases of NMSC arise each year ( $\sim 300$ cases per 100,000 individuals) (39). BCC accounts for $\sim 80 \%$ of skin cancer with a lifetime risk of about 28-30\% (40). BCC is locally invasive and destructive, but has a low metastatic potential of $0.0028-0.55 \%$ (41). SCC accounts for $\sim 20 \%$ of skin cancers with a lifetime risk of 7-11\% $(42,43)$. Compared with BCC, it has a much higher and more variable metastatic potential of 3.6\%-30\%, depending on the site and etiology of the lesion (44). It has been suggested that all SCC are derived from precursor lesions (i.e.; AK); although only $0.1-10 \%$ of AK lesions are known to progress to SCC (45-47). 
AK has a prevalence ranging from $11 \%$ to as high as $80 \%$ in different populations and age groups $(45,48)$. SCC in situ progresses to invasive SCC in only a small number of cases (2-5\%) and demonstrates a low metastatic potential $(49,50)$. The incidence of SCC in situ can vary from 14.9 to 142 per 100,000 individuals, depending on the ethnic population studied (51). Keratoacanthoma (KA) has been classified as either a distinct lesion or a subtype of SCC, and its reported incidence varies from 7 to 104 per 100,000 (52). KA lesions are commonly solitary, demonstrate phases of growth, maturation and spontaneous regression, and rarely metastasize (44). Given the differing metastatic capabilities of NMSC lesions, the degree of chromosomal instability between each of the lesions would also expected to vary.

Jin et al. $(53,54)$ investigated 69 new and previously published short-term (5-10 days) BCC cultures and identified recurrent numerical aberrations of +18 (30\%), +7, +X (17\%) and +9 (14\%), structural rearrangements involving 9q (24\%), and breakpoints involving 1p32, 1p22, 1q11, 1q21, 4q21 and 4q31 (10\%). Other studies have also found structural abnormalities in BCC, such as translocations and inversions involving 9q $(7,55,56)$. Casalone et al. (57) studied 73 BCC samples, harvested within 24 hours, as well as short-term cultures (10-28 days). Trisomy of chromosome 6 was found to be the most recurrent change; only observed in a small number of the samples harvested within 24 hours (57). FISH analysis detected this variation in additional samples, but not in any of the short-term cultures, suggesting that the use of such cultures may lead to erroneous data (57). CGH analysis of 15 BCC samples identified recurrent CNV, such as gain of 6p (47\%), 6q, 9p (20\%), 7 and X (13\%), as well as loss of 9q (33\%) (58). Follow-up LOH analysis determined loss of 9q22.3 in 53\% of cases (58). Other studies have found LOH 
at 9q22 (46-60\%), 9p21-p22 (55\%), 17q21 (34\%), 1q (14\%), and 17p13 (11\%) in BCC (59-62). In summary, results obtained from karyotypic, CGH, FISH and LOH analyses indicate that BCC karyotypes typically consist of only 1-3 aberrations $(53,54)$, with trisomy $6(57,58)$ and loss of the 9q region $(58-61)$ being the primary numerical aberrations. Overall, cytogenetic analyses indicate that BCC is genetically relatively stable compared to other tumors, which may be reflected in its low metastatic capability. Figure 4-2 summarizes recurrent aberrations $(>10 \%)$ detected in BCC samples by karyotypic, CGH, FISH and LOH techniques.

Jin et al. (63) also identified recurrent numerical aberrations in short-term cultures from 13 primary cutaneous SCC and 10 previously published cases. These included gains of 7p (32\%) and 8q (27\%), and losses of 21 (41\%), 8p (36\%), 4p, 11p, Y (32\%), 13, 18q (27\%), 10p, X (23\%) and 9p (18\%) (63). Isochromosomes (defined as a chromosome that has lost one of its arms and replaced it with an exact copy of the other arm) such as i(1p), $\mathrm{i}(1 \mathrm{q}), \mathrm{i}(5 p), \mathrm{i}(8 \mathrm{q}), \mathrm{i}(9 p)$, and i(9q) (all $<20 \%)$, were also represented in these cases, with $i(8 q)$ and $i(9 q)$ believed to be early genetic events (63). By examining direct preparations of three primary cutaneous SCC, Casalone et al. (57) identified aberrations not detected in short-term cultures, including $-1,+6,+8,+9,+11,-14,+16$ and +21 ; although due to a limited number of samples, these could not be considered recurrent. Using CGH, gains of 3q (47\%), 17q (40\%), 14q, Xq (33\%), 4p, 8q (27\%), 1q, 5p, 7q, 9q, 10q and 20q (20\%), and losses of 3p (53\%), 18q (47\%), 17p (33\%), 4q (27\%), 5q, 8p, 11p, 13q and 18p (20\%) were identified in a study of 14 SCC, including five arising from an adjacent AK (64). Although many of the CNVs were shared between SCC and AK, loss of 18q was specific to SCC $(\mathrm{P}$ value $=0.04)$ and may indicate an important region relating to 
malignant progression (64). Another study investigated five SCC cell lines and identified recurrent gains on 11q (100\%), 7p (60\%) and 8q (60\%), and recurrent losses on $3(80 \%)$, 9p (80\%) and 8p (60\%) (65). LOH analysis of SCC (including in situ lesions) has revealed frequent loss of 17q (43\%), 13q (38\%), 17p (34\%), 9p (32\%), 3p (26\%) and 2q (20\%) (59). Recurrent aberrations associated with SCC (>10\%), as detected by karyotypic, CGH and LOH analysis can be seen in Figure 4-3.

Very few cytogenetic studies have been performed on other keratinocyte-derived skin tumors, but do indicate some shared cytogenetic features between premalignant and malignant lesions. These include: (i) gains of chromosome 7 and 20 and structural rearrangements involving chromosomes 1 and 4, identified by short-term cultures of three AK and two SCC in situ lesions (66); (ii) gain of 3q, 4p, 17q (33\%), 5p, 9q and 17p (25\%) and loss of 9p, 13q (53\%), 3p, 4q, 11p and 17p (25\%) identified by CGH analysis of AK (64); (iii) a similar but higher frequency of LOH in AK compared to SCC, including loss at 17p (64\%), 13q (52\%), 17q (46\%), 9p (39\%), 9q (22\%) and 3p (31\%) (67); and (iv) recurrent loss of TP53 (27\%) in SCC in situ (68). LOH of the region encoding CDKN2A in both SCC (46\%) and AK (21\%) (69), in addition to rearrangements involving 3p13 in AK and SCC (66), indicate that these aberrations might be early genetic events that contribute to malignant potential. Furthermore, CGH analysis has detected gain of $7 \mathrm{p}$ in metastatic SCC lesions, suggesting EGFR as a candidate gene conferring metastatic potential (70). Cytogenetic abnormalities associated with KA include: (i) alteration of 2p13, identified by karyotypic analysis in two cases (71,72); (ii) gains of 8q (20\%), 1p and 9q (16\%), and losses of 3p, 9p, 19p (20\%), and 19q (16\%) detected by CGH analysis of lesions mostly derived from immunosuppressed organ 
transplant recipients (73); and (iii) rare $\mathrm{LOH}$, with isolated cases of loss detected at 9p, 9q, and 10q (74). Clausen et al. (75) detected significant differences in the frequency of copy number aberrations between SCC and KA, including more frequent gain of 1p, 14q, $16 q, 20 q$ and loss of $4 p$ in SCC $(P \leq 0.03)$, as well as more frequent loss of 9p in KA (P = 0.04), providing further evidence to the theory that SCC and KA are distinct forms of NMSC. Overall, cytogenetic studies suggest that both AK and SCC are more genetically unstable than BCC and KA.

\section{Rare Cancers of the Skin}

Merkel cell carcinoma (MCC) is an aggressive neuroendocrine tumor of the skin, affecting about 1,000 individuals in the United States each year ( 3.3 per 100,000) (76,77). Karyotyping of six MCC cases revealed various structural and numerical rearrangements of chromosome 1 and numerical aberrations, including -13 (67\%), +11 and -22 (33\%) (78). CGH analysis has identified recurrent numerical aberrations, including +19q (63\%), +19p (50\%), +1p (54\%), -3p (46\%), +1q, +X (42\%), +5p, +8q (38\%), -10, +3q, -13q (33\%), +20p (29\%), +7p, -17p, +20q (25\%), -5q, +6q, +7q, -8p, $+13 q,+18 q(21 \%),-11 q$ and $+21(17 \%)$ in MCC (77). In addition, the average number of imbalances was noted to be different in patients surviving $>24$ months (6.6) compared with $<24$ months (11.2) (77). In another study, CGH analysis of 19 cases revealed many of the same recurrent aberrations, including +6 (42\%), +1q11-q31, +5p (32\%), +1q32-qter (26\%), +1p33-pter, +12, -13q13-q31 (21\%) and -4q (16\%) (89). FISH analysis of 10 cutaneous MCC lesions has also confirmed +6 in $60 \%$ of cases (80). Two independent LOH studies have identified deletion of 10q23 (43\% of cases), although this 
is unlikely to involve PTEN (81), in addition to deletion of 1p35-36 (70\% of cases) (82).

Dermatofibrosarcoma protuberans (DFSP) is a rare cutaneous mesenchymal tumor which arises from spindle cells within the dermis $(83,84)$. The tumor slowly infiltrates adjacent and deeper subcutaneous tissues, and shows a low propensity for metastases $(83,84)$. The annual incidence of DFSP is estimated at 4.2 per million people in the United States (83). The tumor is associated with a signature reciprocal translocation involving chromosomes 17 and 22 [t(17;22)(q22;q13)], with fusion of the COL1A1 and PDGFB genes $(84,85)$. This translocation involves the formation of supernumerary ring chromosomes in about $70 \%$ of cases $(84,66)$. CGH analyses of DFSP have revealed consistent recurrent aberrations, including 11 cases showing $+17 q 21$-qter (100\%), +22pter-q13 (82\%), and +5 (27\%) (87); and 12 cases showing aberrations of +17q22-qter (83\%), +22q13 (75\%), and 8q24.1-qter (25\%) (88).

Cutaneous lymphomas, including cutaneous T-cell lymphoma (CTCL) and cutaneous B-cell lymphoma (CBCL) subtypes, affect approximately 3 in 1,000,000 people annually (21). CTCL is the more common of the two subtypes, affecting about 1,500 individuals annually (89), and includes mycosis fungoides (MF) and Sezary syndrome (SS) (90). CBCL accounts for only 10-25\% of cutaneous lymphomas (21) and includes follicular lymphoma (FL), marginal zone B-cell lymphoma and large B-cell lymphoma subtypes (91). Karyotypic testing of 18 SS lesions revealed structural aberrations of chromosomes 10, 17 (28\%), 1p (22\%), 6q, and 14q (17\%) (92). FISH analysis verified rearrangements of 1p, 17p (33\%), and 10 (27\%) (92). CGH analysis of MF lesions has revealed recurrent numerical aberrations of 1p (38\%), $-17 p(21 \%),+4 / 4 q$ (18\%), $-10 q / 10,+18,-19(15 \%)$, and $+17 q / 17$ (12\%) (92). A review of 166 SS samples 
revealed recurrent losses of chromosomes $1,2,6 q, 9,10,13,16$ and 17, as well as structural rearrangement of 17q (93). In another study, CGH analysis of three MF and four SS cases identified recurrent copy number changes of $-10 \mathrm{q} 23$ (29\%) and $+17 \mathrm{q} 11.2$ (71\%), suggesting that the duplication at $17 q$ seen in both MF and SS may be an early clonal event (94). LOH analysis has detected recurrent deletions at 9p (46\%), 17p (42\%), 10q and 2p (14\%) in 15 cases of SS, and at 9p (16\%), 10q (12\%), 1p and 17p (10\%) in 51 cases of MF (90).

CGH analysis of CBCL subtypes has identified: (i) recurrent numerical alterations of $+2 q,+7 q,+12,+13,-17 p,+18$ and -19 in nine cases of primary cutaneous large B-cell lymphoma lesions; (ii) a sole deletion of $-17 p(60 \%)$ in five secondary lesions; and (iii) aberrations of $+3 q,+4$ and $+7 q$ in four cases of primary cutaneous FL (95). In another study, FISH analysis of 27 cases of FL identified recurrent translocations involving the immunoglobulin heavy chain gene (IGH) on chromosome 14 (52\%) (96). These were associated with BCL2 [t(14:18)(q32:q31)] in 41\% of cases and BCL6 [t(3:14)(q27:q32)] in $7 \%$ of cases (96). FISH analysis of 14 cases of primary cutaneous large B-cell lymphoma also showed translocations involving IGH (50\%); associated with MYC [t(8:14)(q24:q32)] in 36\% of cases and BCL6 [t(3:14)(q27:q32) in 14\% of cases (97). Translocations involving MYC and BCL6, independent of the IGH locus, were detected in an additional $7 \%$ and $21 \%$ of lesions, respectively (97).

\section{Recent Advances in Cytogenetic Detection Methods}

While cytogenetic methods have predominantly been employed as research tools for the genetic characterization of diseases, recent advances in these technologies has prompted 
interest in their use in the diagnostic setting. However, their routine clinical implementation has been hampered by high cost, prolonged turnaround time, and the expertise required for most cytogenetic detection methods. Nonetheless, efforts have been undertaken to increase their application by reducing time and cost, and increasing the convenience and automation of these methods.

Array-based CGH (aCGH) utilizes microarray chips dotted with thousands of positionally-defined specific probes, to which differentially labeled normal and tumor DNA are co-hybridized, and scanned using computerized fluorescence imaging and analysis systems (14). This has allowed CGH resolution to vastly improve, from $>5-20$ mb (for traditional CGH) to <100 kbp, with some high-resolution tiling arrays (HR-CGH) now able to resolve to 50-200 bp (98). In addition, single nucleotide polymorphism (SNP)-based CNV arrays can detect microscopic CNVs, polyploidy, mosaicisms and uniparental disomy $(99,100)$. A number of studies using aCGH and CNV-arrays have been performed on skin cancers. These techniques have confirmed and better resolved many of the genomic aberrations seen in previous traditional CGH and LOH studies, as well as identifying novel alterations. Next-generation sequencing has recently been implemented as a cytogenetic tool, in an effort to more easily detect structural abnormalities, such as translocations and inversions (16). However, as a result of current costs, it has not yet been used widely and no studies to date have utilized this methodology to detect genomic aberrations in skin cancers.

aCGH analysis of five cell lines derived from different melanoma metastases in a single patient identified a primary cluster of genomic imbalances, including $+3 p,+7,-9 p$, $-10 p,-14 q,-16 q,-17 p$ and $+17 q(101)$. Another study, investigating two formalin-fixed 
paraffin-embedded (FFPE) melanoma samples, confirmed known aberrations of $+1 \mathrm{q}$ and -9p (102). More recently, two large aCGH studies of melanoma samples (126 and 102 samples; 80 shared cases) were undertaken $(103,104)$. In these reports, Curtin et al. $(103,104)$ aimed to investigate possible differences in chromosomal aberrations between tumors derived from different sites or associated with sun-induced damage. Amongst the different subtypes of melanoma (i.e.; those with chronic sun-induced damage; those without chronic sun-induced damage; and at mucosal and acral sites), gains of $6 \mathrm{p}, 17 \mathrm{q}$ and 20q, as well as loss of 9p and 21q, are common aberrations (104). Gain involving the CCND1 locus (11q13) and regions of chromosome 22, and loss of 4q, are significantly more common in tumors associated with chronic sun-induced damage $(\mathrm{p}=0.001,0.004$ and 0.004 , respectively) (104), suggesting that these aberrations may arise as a result of long term exposure to ultraviolet radiation. Copy number amplifications of $4 \mathrm{q}$ are shared between lesions, both with and without chronic sun-induced damage, and appear to involve the KIT oncogene (which is essential for melanocyte survival and development) (103). CNV-array analysis of 76 melanoma cell lines detected: (i) frequent LOH at 6q, 9p, 9q, 10p, 10q, 11q and 17p; (ii) 174 homozygous deletions, primarily associated with CDKN2A, PTEN, PTPRD and HDAC4; and (iii) 197 focal amplifications, many of which were associated with BRAF, CCND1, MDM2, MITF, NRAS and PIK3CA (99).

Ma et al. (105) detected loss of the CSMD1 (8p23) tumor suppressor gene in SCC (29\%) and BCC (17\%) using aCGH. In an effort to support the applicability of aCGH in the clinical setting, a study on archival FFPE SCC, SCC precursors and normal skin has also been undertaken (106). The most common aberration detected was loss of 10q-ter, containing the INPP5A (10q26.3) tumor suppressor gene, which was found in both SCC 
in situ and SCC, but not in non-tumorous sun-damaged skin (106). CNV-array analysis of 16 primary SCC and 2 metastatic SCC identified common LOH at 2q, 3p, 8p, 9p and 13, as well as gains at $3 \mathrm{q}$ and $8 \mathrm{q}$ (107). Analysis of paired primary and metastatic SCC (to lymph nodes) from two of these patients suggested that the primary and metastatic lesions shared common genetic aberrations (although these were different for the two patients), and that the acquisition of additional genetic aberrations is associated with metastatic spread in SCC (107). LOH at 9q21-q31 was detected in 93\% of BCC (108), suggesting a much higher rate of $9 q$ loss than previously detected with other techniques (58-61). In addition, loss of a novel region, 6q23-q27, was identified in 36\% of BCC (108). These studies support CNV-arrays as a powerful tool for the identification of novel cytogenetic aberrations.

aCGH has also been used to investigate MCC, DFSP and MF lesions. In a study of 25 MCC samples, oligonucleotide aCGH was used to detect recurrent aberrations of $+1,-3 p,+3 q,-4,+5 p,-5 q,+6,-7,-10$ and -13 (109). In addition, increasing numbers of genomic aberrations correlated with decreased patient survival $(\mathrm{p}=0.04)(109)$. Another aCGH study of 10 cases of MCC detected recurrent aberrations, including $+1 \mathrm{q},+6 \mathrm{p},+11$ and -17p (110). In the case of DFSP, aCGH analysis using a pooled approach identified recurrent copy number gains of +8q24.3, +17q21.33-qter and +22cen-q13.1 (84). Finally, oligonucleotide aCGH detected common chromosomal imbalances of -6q21.3, +7q33.3-q35, +8q24.21, -9p21.3, -9q31.2, +9q34-qter, -10p11.22, +10p14, -13q14.11, -16q23.2, -16q24, -17p13.1 and +17q21.1 in 41 skin biopsies from MF patients (111). Interestingly, aberrations at 8q24.21,9p21.3 and 10q26-qter correlated with overall patient survival ( $\mathrm{p}=0.017,0.042$ and 0.022 , respectively) (111). 


\section{Implications of Cytogenetic Findings}

The employment of cytogenetic methods, in concert with other molecular techniques (outlined in Chapter 3), has greatly increased our ability to identify putative genes or genetic regions associated with tumorigenesis. Genomic aberrations associated with skin tumors include those that are shared amongst a number of different lesions and those that are distinct to a particular lesion or even present at a specific stage of skin cancer development. In addition, the degree of genomic instability appears to be a measure of the potential aggressiveness of a tumor.

A number of recurrent genomic aberrations are shared between different forms of skin cancer, including rearrangements and numerical abnormalities of chromosome 1 (melanoma, BCC, SCC, AK, KA, MCC, DFSP, CTCL); -3p (SCC, AK, KA, MCC); +3q (SCC, AK, MCC, CBCL); trisomy of all or part of 6 (melanoma, BCC, SCC, MCC); trisomy of all or part of 7 (melanoma, BCC, SCC, AK, MCC and CBCL); +8q (melanoma, SCC, KA, MCC, DFSP); -9p (dysplastic nevi, melanoma, BCC, SCC, AK, KA, CTCL); +9q (melanoma, SCC, AK, KA); loss of part or all of 10 (melanoma, SCC, MCC, CTCL); -17p (melanoma, BCC, SCC, SCC in situ, AK, MCC, CTCL, CBCL); +17q (melanoma, SCC, AK, DFSP); and gain of all or part of 20 (melanoma, SCC, AK, MCC, DFSP). Within these regions, a number of potential tumor suppressor genes and oncogenes exist, including: (i) FHIT (3p14.2), which is involved in cell cycle control (112) and whose loss has been associated with MCC (77) and many other malignancies (112); (ii) BCL6 (3q27), a breakpoint region implicated in cutaneous lymphomas $(96,97)$, that mediates transcriptional repression (113); (iii) E2F3 (6p22) that is an essential 
component of $R B$ - and $M Y C$-mediated cell cycle progression pathways $(114,115)$ and whose over-expression is seen in retinoblastoma and bladder cancer (116); (iv) CDK6 (7q21-q22), involved in $R B$-mediated cell cycle progression (117), and NRCAM (7q31.1-q31.2), both of which are over-expressed in melanoma cell lines (118); (v) MYC (8q24.12-q24.13), which promotes cell proliferation (115) and is known to further destabilize the genome (119,120); (vi) CDKN2A (9p21), which encodes two major proteins p16(INK4A) and p14(ARF) that regulate the $R B$ and $P 53$ cell cycle progression pathways, respectively [for a review see (121)]; (vii) PTEN (10q23.31), which suppresses tumorigenicity by blocking cell cycle progression (122) and whose loss is seen in various human cancers (123); (viii) TP53 (17p13.1), which is disrupted in about 50\%-60\% of all human cancers $(124,125)$ and plays a role in multiple cellular functions, including regulation of cell cycle arrest, apoptosis, glycolysis, autophagy, oxidative stress, invasion, motility, angiogenesis and differentiation [for a review see (126)]; and (ix) E2F1 (20q11.2), an essential component of $R B$-mediated cell cycle progression and MYC-induced apoptosis $(114,115)$.

It is also evident that amplifications and deletions in some regions are detected at similar frequencies within the same types of skin lesions, including chromosomes 9q, 15, 18, 19, 21 and 22 in melanoma and $4 q$ and $9 q$ in AK. Some of these changes may reflect background genomic instability rather than being directly associated with tumorigenesis. Additionally, it has also been shown that regions of amplification contain genes that are both over- and under-expressed, indicating that chromosomal aberrations do not always reflect changes at the genetic level (127).

Some aberrations are found to be more prominent in specific skin cancers. For 
example, loss of 18q in SCC as compared to AK. 18q harbors potential candidate genes SMAD2 (18q21) and SMAD4 (18q21.1) that mediate TGF-beta signaling and cell growth regulation (128), and which have been implicated in malignant progression in other non-cutaneous tumors $(129,130)$. Another example is loss of 9q22 in sporadic BCC. 9q22 contains the PTCH1 (9q22.3) tumor suppressor gene that is part of the hedgehog developmental signaling pathway, which has been implicated in the pathogenesis of hereditary BCC (131). In DFSP, translocation involving 17q22 and 22q13 creates a gene fusion between COL1A1 (17q21.31-q22) and PDGFB (22q12.3-13.1), resulting in aberrant expression of $P D G F B$ (84) which promotes cellular proliferation, disorganized growth and inhibits apoptosis $(132,133)$. Other studies have implicated aberrations in specific regions as early events in skin tumor development, such as loss of 9p (CDKN2A locus), which has been detected in dysplastic nevi, primary and metastatic melanoma $(19,27,28,33-35)$, as well as in SCC and AK (69).

Cytogenetic analysis is a powerful means to detect aberrant chromosomal regions that may harbor tumor suppressor genes and oncogenes. Analyses of melanoma and NMSC have identified a number of consistently aberrant regions. These regions contain genes, which when altered have been implicated in cancer pathogenesis and progression pathways, including cell growth, signaling, differentiation and apoptosis. In addition, some aberrant regions appear to be specific to only one type of skin tumor, possibly containing genes more relevant to the morphological characteristics and/or biologic behavior of that particular skin tumor. The implementation of newer cytogenetic techniques with higher resolutions of detection will continue to improve our understanding of the genetic basis of skin tumors. 


\section{REFERENCES}

1. Ford CE. Human cytogenetics: its present place and future possibilities. Am J Hum Genet. 1960;12:104-117.

2. Glassman AB. Cytogenetics, in situ hybridization and molecular approaches in the diagnosis of cancer. Ann Clin Lab Sci. 1998;28:324-330.

3. Haferlach C, Bacher U, Tiu R, et al. Myelodysplastic syndromes with del(5q): indications and strategies for cytogenetic testing. Cancer Genet Cytogenet. 2008;187:101-11.

4. Folpe AL, Goldblum JR, Rubin BP, et al. Morphologic and immunophenotypic diversity in Ewing family tumors: a study of 66 genetically confirmed cases. Am J Surg Pathol 2005;29:1025-1033.

5. Nowell P, Hungerford DA. A minute chromosome in human chronic granulocytic leukemia. Science 1960;132:1497.

6. Rowley JD. Letter: A new consistent chromosomal abnormality in chronic myelogenous leukaemia identified by quinacrine fluorescence and Giemsa staining. Nature 1973;243:290-293.

7. Mertens F, Heim S, Mandahl N, et al. Cytogenetic analysis of 33 basal cell carcinomas. Cancer Res. 1991;51:954-957.

8. Lotem M, Yehuda-Gafni O, Butnaryu E, et al. Cytogenetic analysis of melanoma cell lines: subclone selection in long-term melanoma cell cultures. Cancer Genet Cytogenet. 2003;142:87-91.

9. Kallioniemi OP, Kallioniemi A, Piper J, et al. Optimizing comparative genomic hybridization for analysis of DNA sequence copy number changes in solid tumors. 
Genes Chromosomes Cancer. 1994;10:231-243.

10. A Kallioniemi, T Visakorpi, R Karhu, D Pinkel and OP Kallioniemi. (1996). Gene Copy Number Analysis by Fluorescence in Situ Hybridization and Comparative Genomic Hybridization. Methods 9(1), 113-21.

11. Thompson CT and Gray JW. Cytogenetic profiling using fluorescence in situ hybridization (FISH) and comparative genomic hybridization (CGH). J Cell Biochem Suppl. 1993;17G:139-143.

12. Varella-Garcia M. Molecular cytogenetics in solid tumors: laboratorial tool for diagnosis, prognosis, and therapy. Oncologist. 2003;8:45-58.

13. Happle R. Loss of heterozygosity in human skin. J Am Acad Dermatol. 1999;41:143-164.

14. Pinkel D, Segraves R, Sudar D, et al. High resolution analysis of DNA copy number variation using comparative genomic hybridization to microarrays. Nat Genet. 1998;20:207-211.

15. Redon R, Ishikawa S, Fitch KR, et al. Global variation in copy number in the human genome. Nature. 2006;444:444-454.

16. Chen W, Kalscheuer V, Tzschach A, et al. Mapping translocation breakpoints by next-generation sequencing. Genome Res. 2008;18:1143-1149.

17. Knutsen T, Gobu V, Knaus R, et al. The interactive online SKY/M-FISH \& CGH database and the Entrez cancer chromosomes search database: linkage of chromosomal aberrations with the genome sequence. Genes Chromosomes Cancer. 2005;44:52-64.

18. Mitelman Database of Chromosome Aberrations in Cancer. Mitelman F, 
Johansson B and Mertens F (Eds.). 2009. Available from: http://cgap.nci.nih.gov/Chromosomes/Mitelman.

19. Dorkeld F, Bernheim A, Dessen P and Huret JL. A database on cytogenetics in haematology and oncology. Nucleic Acids Res. 1999;27:353-354. Available from: http://atlasgeneticsoncology.org/.

20. Weinstock MA. Epidemiology of nonmelanoma skin cancer: clinical issues, definitions, and classification. J Invest Dermatol. 1994;102:4S-5S.

21. Burg G and Kempf W. Cutaneous B-cell lymphoma. eMedicine 2008. Available from: http://emedicine.medscape.com/article/1099540-overview.

22. American Cancer Society. Cancer facts and figures. Atlanta: American Cancer Society 2009. Available from: http://www.cancer.org/downloads/STT /500809web.pdf.

23. Hoglund M, Gisselsson D, Hansen GB, et al. Dissecting karyotypic patterns in malignant melanomas: temporal clustering of losses and gains in melanoma karyotypic evolution. Int J Cancer. 2004;108:57-65.

24. Bastian BC, Olshen AB, LeBoit PE, et al. Classifying melanocytic tumors based on DNA copy number changes. Am J Pathol. 2003;163:1765-1770.

25. Richmond A, Fine R, Murray D, et al. Growth factor and cytogenetic abnormalities in cultured nevi and malignant melanomas. J Invest Dermatol. 1986;86:295-302.

26. Marras S, Faa G, Dettori T, et al. Chromosomal changes in dysplastic nevi. Cancer Genet Cytogenet 1999;113:177-9.

27. Cowan JM and Francke U. Cytogenetic analysis in melanoma and nevi. Cancer 
Treat Res. 1991;54:3-16.

28. Balazs M, Adam Z, Treszl A, et al. Chromosomal imbalances in primary and metastatic melanomas revealed by comparative genomic hybridization. Cytometry. 2001;46:222-232.

29. Barks JH, Thompson FH, Taetle R, et al. Increased chromosome 20 copy number detected by fluorescence in situ hybridization (FISH) in malignant melanoma. Genes Chromosomes Cancer. 1997;19:278-285.

30. Treszl A, Adany R, Rakosy Z, et al. Extra copies of c-myc are more pronounced in nodular melanomas than in superficial spreading melanomas as revealed by fluorescence in situ hybridisation. Cytometry B Clin Cytom. 2004;60:37-46.

31. Matsuta M, Imamura Y, Matsuta M, et al. Detection of numerical chromosomal aberrations in malignant melanomas using fluorescence in situ hybridization. J Cutan Pathol. 1997;24:201-205.

32. Rakosy Z, Vizkeleti L, Ecsedi S, et al. EGFR gene copy number alterations in primary cutaneous malignant melanomas are associated with poor prognosis. Int $\mathrm{J}$ Cancer. 2007;121:1729-1737.

33. Hussein MR, Roggero E, Tuthill RJ, et al. Identification of novel deletion Loci at 1p36 and 9p22-21 in melanocytic dysplastic nevi and cutaneous malignant melanomas. Arch Dermatol. 2003;139:816-817.

34. Uribe P, Wistuba, II and Gonzalez S. Allelotyping, microsatellite instability, and BRAF mutation analyses in common and atypical melanocytic nevi and primary cutaneous melanomas. Am J Dermatopathol. 2009;31:354-363. 
35. Hussein MR, Sun M, Roggero E, et al. Loss of heterozygosity, microsatellite instability, and mismatch repair protein alterations in the radial growth phase of cutaneous malignant melanomas. Mol Carcinog. 2002;34:35-44.

36. Udart M, Utikal J, Krahn GM, et al. Chromosome 7 aneusomy. A marker for metastatic melanoma? Expression of the epidermal growth factor receptor gene and chromosome 7 aneusomy in nevi, primary malignant melanomas and metastases. Neoplasia. 2001;3:245-254.

37. Utikal J, Udart M, Leiter U, et al. Additional Cyclin D(1) gene copies associated with chromosome 11 aberrations in cutaneous malignant melanoma. Int J Oncol. 2005;26:597-605.

38. Krzywinski M, Schein J, Birol I, et al. Circos: an information aesthetic for comparative genomics. Genome Res 2009;19:1639-1645.

39. American Cancer Society. Overview: Skin cancer - basal and squamous cell. How many people get basal and squamous cell skin cancers? 2008. Available from: http://www.cancer.org/docroot/CRI/content/CRI_2_2_1X_How_many_people_ge t_nonmelanoma_skin_cancer_51.asp?sitearea $=$.

40. Miller DL and Weinstock MA. Nonmelanoma skin cancer in the United States: incidence. J Am Acad Dermatol. 1994;30:774-778.

41. Wong CS, Strange RC and Lear JT. Basal cell carcinoma. BMJ. 2003;327:794-798.

42. Bernstein SC, Lim KK, Brodland DG, et al. The many faces of squamous cell carcinoma. Dermatol Surg. 1996;22:243-254. 
43. Diepgen TL and Mahler V. The epidemiology of skin cancer. Br J Dermatol. 2002;146 Suppl 61:1-6.

44. Skidmore RA, Jr. and Flowers FP. Nonmelanoma skin cancer. Med Clin North Am. 1998;82:1309-1323, vi.

45. Salasche SJ. Epidemiology of actinic keratoses and squamous cell carcinoma. J Am Acad Dermatol. 2000;42:4-7.

46. Marks R, Rennie G and Selwood TS. Malignant transformation of solar keratoses to squamous cell carcinoma. Lancet. 1988;1:795-797.

47. Evans C and Cockerell CJ. Actinic keratosis: time to call a spade a spade. South Med J. 2000;93:734-736.

48. Frost C, Williams G and Green A. High incidence and regression rates of solar keratoses in a queensland community. J Invest Dermatol. 2000;115:273-277.

49. Ramrakha-Jones VS and Herd RM. Treating Bowen's disease: a cost-minimization study. Br J Dermatol. 2003;148:1167-1172.

50. Cohen PR. Bowen's disease: squamous cell carcinoma in situ. Am Fam Physician. 1991;44:1325-1329.

51. Reizner G, Chuang T, Elpern D, et al. Bowen's disease (squamous cell carcinoma in situ) in Kauai, Hawaii. A population-based incidence report. J Am Acad Dermatol. 1994;31:596-600.

52. Reizner GT, Chuang TY, Elpern DJ, et al. Keratoacanthoma in Japanese Hawaiians in Kauai, Hawaii. Int J Dermatol. 1995;34:851-853.

53. Jin Y, Mertens F, Persson B, et al. Nonrandom numerical chromosome abnormalities in basal cell carcinomas. Cancer Genet Cytogenet. 1998;103:35-42. 
54. Jin Y, Martins C, Salemark L, et al. Nonrandom karyotypic features in basal cell carcinomas of the skin. Cancer Genet Cytogenet. 2001;131:109-119.

55. Jin Y, Merterns F, Persson B, et al. The reciprocal translocation t(9;16)(q22;p13) is a primary chromosome abnormality in basal cell carcinomas. Cancer Res. 1997;57:404-406.

56. Kawasaki-Oyama RS, Andre FS, Caldeira LF, et al. Cytogenetic findings in two basal cell carcinomas. Cancer Genet Cytogenet. 1994;73:152-156.

57. Casalone R, Mazzola D, Righi R, et al. Cytogenetic and interphase FISH analyses of 73 basal cell and three squamous cell carcinomas: different findings in direct preparations and short-term cell cultures. Cancer Genet Cytogenet. 2000;118:136-143.

58. Ashton KJ, Weinstein SR, Maguire DJ, et al. Molecular cytogenetic analysis of basal cell carcinoma DNA using comparative genomic hybridization. J Invest Dermatol. 2001;117:683-686.

59. Quinn AG, Sikkink S and Rees JL. Basal cell carcinomas and squamous cell carcinomas of human skin show distinct patterns of chromosome loss. Cancer Res. 1994;54:4756-4759.

60. Shen T, Park WS, Boni R, et al. Detection of loss of heterozygosity on chromosome 9q22.3 in microdissected sporadic basal cell carcinoma. Hum Pathol. 1999;30:284-287.

61. Shanley SM, Dawkins H, Wainwright BJ, et al. Fine deletion mapping on the long arm of chromosome 9 in sporadic and familial basal cell carcinomas. Hum Mol Genet. 1995;4:129-133. 
62. Saridaki Z, Koumantaki E, Liloglou T, et al. High frequency of loss of heterozygosity on chromosome region 9p21-p22 but lack of p16INK4a/p19ARF mutations in Greek patients with basal cell carcinoma of the skin. J Invest Dermatol. 2000;115:719-725.

63. Jin Y, Martins C, Jin C, et al. Nonrandom karyotypic features in squamous cell carcinomas of the skin. Genes Chromosomes Cancer. 1999;26:295-303.

64. Ashton KJ, Weinstein SR, Maguire DJ, et al. Chromosomal aberrations in squamous cell carcinoma and solar keratoses revealed by comparative genomic hybridization. Arch Dermatol. 2003;139:876-882.

65. Popp S, Waltering S, Herbst C, et al. UV-B-type mutations and chromosomal imbalances indicate common pathways for the development of Merkel and skin squamous cell carcinomas. Int J Cancer. 2002;99:352-360.

66. Jin Y, Jin C, Salemark L, et al. Clonal chromosome abnormalities in premalignant lesions of the skin. Cancer Genet Cytogenet. 2002;136:48-52.

67. Rehman I, Takata M, Wu YY, et al. Genetic change in actinic keratoses. Oncogene. 1996;12:2483-2490.

68. Lee HJ, Kim JS, Ha SJ, et al. p53 gene mutations in Bowen's disease in Koreans: clustering in exon 5 and multiple mutations. Cancer Lett. 2000;158:27-33.

69. Mortier L, Marchetti P, Delaporte E, et al. Progression of actinic keratosis to squamous cell carcinoma of the skin correlates with deletion of the 9p21 region encoding the p16(INK4a) tumor suppressor. Cancer Lett. 2002;176:205-214. 
70. Shimizu T, Izumi $\mathrm{H}$, Oga A, et al. Epidermal growth factor receptor overexpression and genetic aberrations in metastatic squamous-cell carcinoma of the skin. Dermatology. 2001;202:203-206.

71. Kim DK, Kim JY, Kim HT, et al. A specific chromosome aberration in a keratoacanthoma. Cancer Genet Cytogenet. 2003;142:70-72.

72. Mertens F, Heim S, Mandahl N, et al. Clonal chromosome aberrations in a keratoacanthoma and a basal cell papilloma. Cancer Genet Cytogenet. 1989;39:227-232.

73. Clausen OP, Beigi M, Bolund L, et al. Keratoacanthomas frequently show chromosomal aberrations as assessed by comparative genomic hybridization. $\mathrm{J}$ Invest Dermatol. 2002;119:1367-1372.

74. Waring AJ, Takata M, Rehman I, et al. Loss of heterozygosity analysis of keratoacanthoma reveals multiple differences from cutaneous squamous cell carcinoma. Br J Cancer. 1996;73:649-653.

75. Clausen OP, Aass HC, Beigi M, et al. Are keratoacanthomas variants of squamous cell carcinomas? A comparison of chromosomal aberrations by comparative genomic hybridization. J Invest Dermatol. 2006;126:2308-2315.

76. Merkel cell carcinoma, information for patients and their physicians. What is Merkel cell carcinoma. 2009. Available from: http://www.merkelcell.org /aboutDisease/index.php.

77. Van Gele M, Speleman F, Vandesompele J, et al. Characteristic pattern of chromosomal gains and losses in Merkel cell carcinoma detected by comparative genomic hybridization. Cancer Res. 1998;58:1503-1508. 
78. Leonard JH, Leonard P and Kearsley JH. Chromosomes 1, 11, and 13 are frequently involved in karyotypic abnormalities in metastatic Merkel cell carcinoma. Cancer Genet Cytogenet. 1993;67:65-70.

79. Larramendy ML, Koljonen V, Bohling T, et al. Recurrent DNA copy number changes revealed by comparative genomic hybridization in primary Merkel cell carcinomas. Mod Pathol. 2004;17:561-567.

80. Vasuri F, Magrini E, Foschini MP, et al. Trisomy of chromosome 6 in Merkel cell carcinoma within lymph nodes. Virchows Arch. 2008;452:559-563.

81. Van Gele M, Leonard JH, Van Roy N, et al. Frequent allelic loss at 10q23 but low incidence of PTEN mutations in Merkel cell carcinoma. Int $\mathrm{J}$ Cancer. 2001;92:409-413.

82. Vortmeyer AO, Merino MJ, Boni R, et al. Genetic changes associated with primary Merkel cell carcinoma. Am J Clin Pathol. 1998;109:565-570.

83. Chen CJ and Siegel DM. Dermatofibrosarcoma protuberans. eMedicine 2009. Available from: http://emedicine.medscape.com/article/1100203-overview.

84. Kaur S, Vauhkonen H, Bohling $\mathrm{T}$, et al. Gene copy number changes in dermatofibrosarcoma protuberans - a fine-resolution study using array comparative genomic hybridization. Cytogenet Genome Res. 2006;115:283-288.

85. Pedeutour F, Simon MP, Minoletti F, et al. Translocation, t(17;22)(q22;q13), in dermatofibrosarcoma protuberans: a new tumor-associated chromosome rearrangement. Cytogenet Cell Genet. 1996;72:171-174. 
86. Pedeutour F, Coindre JM, Sozzi G, et al. Supernumerary ring chromosomes containing chromosome 17 sequences. A specific feature of dermatofibrosarcoma protuberans? Cancer Genet Cytogenet. 1994;76:1-9.

87. Kiuru-Kuhlefelt S, El-Rifai W, Fanburg-Smith J, et al. Concomitant DNA copy number amplification at $17 \mathrm{q}$ and $22 \mathrm{q}$ in dermatofibrosarcoma protuberans. Cytogenet Cell Genet. 2001;92:192-195.

88. Nishio J, Iwasaki H, Ohjimi Y, et al. Overrepresentation of 17q22-qter and 22q13 in dermatofibrosarcoma protuberans but not in dermatofibroma: a comparative genomic hybridization study. Cancer Genet Cytogenet. 2002;132:102-108.

89. The Leukemia and Lymphoma Society. Cutaneous T-cell lymphoma, vol 5. 2006. Available from: http://www.leukemia-lymphoma.org/attachments/National/ br_ 1163608564.pdf.

90. Scarisbrick JJ, Woolford AJ, Russell-Jones R, et al. Allelotyping in mycosis fungoides and Sezary syndrome: common regions of allelic loss identified on 9p, 10q, and 17p. J Invest Dermatol. 2001;117:663-670.

91. Hoefnagel JJ, Dijkman R, Basso K, et al. Distinct types of primary cutaneous large B-cell lymphoma identified by gene expression profiling. Blood. 2005;105:3671-3678.

92. Mao X, Lillington D, Scarisbrick JJ, et al. Molecular cytogenetic analysis of cutaneous T-cell lymphomas: identification of common genetic alterations in Sezary syndrome and mycosis fungoides. Br J Dermatol. 2002;147:464-475. 
93. Mao X, Lillington DM, Czepulkowski B, et al. Molecular cytogenetic characterization of Sezary syndrome. Genes Chromosomes Cancer. 2003;36:250-260.

94. Barba G, Matteucci C, Girolomoni G, et al. Comparative genomic hybridization identifies 17q11.2 approximately q12 duplication as an early event in cutaneous T-cell lymphomas. Cancer Genet Cytogenet. 2008;184:48-51.

95. Gimenez S, Costa C, Espinet B, et al. Comparative genomic hybridization analysis of cutaneous large B-cell lymphomas. Exp Dermatol. 2005;14:883-890.

96. Streubel B, Scheucher B, Valencak J, et al. Molecular cytogenetic evidence of $\mathrm{t}(14 ; 18)(\mathrm{IGH} ; \mathrm{BCL2})$ in a substantial proportion of primary cutaneous follicle center lymphomas. Am J Surg Pathol. 2006;30:529-536.

97. Hallermann C, Kaune KM, Gesk S, et al. Molecular cytogenetic analysis of chromosomal breakpoints in the IGH, MYC, BCL6, and MALT1 gene loci in primary cutaneous B-cell lymphomas. J Invest Dermatol. 2004;123:213-219.

98. Urban AE, Korbel JO, Selzer R, et al. High-resolution mapping of DNA copy alterations in human chromosome 22 using high-density tiling oligonucleotide arrays. Proc Natl Acad Sci USA. 2006;103:4534-4539.

99. Stark M and Hayward N. Genome-wide loss of heterozygosity and copy number analysis in melanoma using high-density single-nucleotide polymorphism arrays. Cancer Res. 2007;67:2632-2642.

100. Gijsbers AC, Lew JY, Bosch CA, et al. A new diagnostic workflow for patients with mental retardation and/or multiple congenital abnormalities: test arrays first. Eur J Hum Genet. 2009;17:1394-1402. 
101. Sabatino M, Zhao Y, Voiculescu S, et al. Conservation of genetic alterations in recurrent melanoma supports the melanoma stem cell hypothesis. Cancer Res. 2008;68:122-131.

102. Harvell JD, Kohler S, Zhu S, et al. High-resolution array-based comparative genomic hybridization for distinguishing paraffin-embedded Spitz nevi and melanomas. Diagn Mol Pathol. 2004;13:22-25.

103. Curtin JA, Busam K, Pinkel D, et al. Somatic activation of KIT in distinct subtypes of melanoma. J Clin Oncol. 2006;24:4340-4346.

104. Curtin JA, Fridlyand J, Kageshita T, et al. Distinct sets of genetic alterations in melanoma. N Engl J Med. 2005;353:2135-2147.

105. Ma C, Quesnelle KM, Sparano A, et al. Characterization CSMD1 in a large set of primary lung, head and neck, breast and skin cancer tissues. Cancer Biol Ther. 2009;8:907-916.

106. Hostetter G, Kim SY, Savage S, et al. Random DNA fragmentation allows detection of single-copy, single-exon alterations of copy number by oligonucleotide array CGH in clinical FFPE samples. Nucleic Acids Res. 2009 Oct 29. [Epub ahead of print]

107. Purdie KJ, Lambert SR, Teh MT, et al. Allelic imbalances and microdeletions affecting the PTPRD gene in cutaneous squamous cell carcinomas detected using single nucleotide polymorphism microarray analysis. Genes Chromosomes Cancer. 2007;46:661-669. 
108. Teh MT, Blaydon D, Chaplin T, et al. Genomewide single nucleotide polymorphism microarray mapping in basal cell carcinomas unveils uniparental disomy as a key somatic event. Cancer Res. 2005;65:8597-8603.

109. Paulson KG, Lemos BD, Feng B, et al. Array-CGH reveals recurrent genomic changes in Merkel cell carcinoma including amplification of L-Myc. J Invest Dermatol. 2009;129:1547-1555.

110. Sastre-Garau X, Peter M, Avril MF, et al. Merkel cell carcinoma of the skin: pathological and molecular evidence for a causative role of MCV in oncogenesis. J Pathol. 2009;218:48-56.

111. Salgado R, Servitje O, Gallardo F, et al. Oligonucleotide Array-CGH Identifies Genomic Subgroups and Prognostic Markers for Tumor Stage Mycosis Fungoides. J Invest Dermatol 2009 Sep 17. [Epub ahead of print]

112. Pekarsky Y, Garrison PN, Palamarchuk A, et al. Fhit is a physiological target of the protein kinase Src. Proc Natl Acad Sci USA. 2004;101:3775-3779.

113. Deweindt C, Albagli O, Bernardin F, et al. The LAZ3/BCL6 oncogene encodes a sequence-specific transcriptional inhibitor: a novel function for the BTB/POZ domain as an autonomous repressing domain. Cell Growth Differ. 1995;6:1495-1503.

114. Nevins JR. Toward an understanding of the functional complexity of the E2F and retinoblastoma families. Cell Growth Differ. 1998;9:585-593.

115. Leone G, Sears R, Huang E, et al. Myc requires distinct E2F activities to induce S phase and apoptosis. Mol Cell. 2001;8:105-113. 
116. Santos GC, Zielenska M, Prasad M, et al. Chromosome 6p amplification and cancer progression. J Clin Pathol. 2007;60:1-7.

117. Meyerson M and Harlow E. Identification of G1 kinase activity for cdk6, a novel cyclin D partner. Mol Cell Biol. 1994;14:2077-2086.

118. Okamoto I, Pirker C, Bilban M, et al. Seven novel and stable translocations associated with oncogenic gene expression in malignant melanoma. Neoplasia. 2005;7:303-311.

119. Felsher DW and Bishop JM. Transient excess of MYC activity can elicit genomic instability and tumorigenesis. Proc Natl Acad Sci USA. 1999;96:3940-3944.

120. Karlsson A, Deb-Basu D, Cherry A, et al. Defective double-strand DNA break repair and chromosomal translocations by MYC overexpression. Proc Natl Acad Sci. U S A 2003;100:9974-9979.

121. Sharpless NE. INK4a/ARF: a multifunctional tumor suppressor locus. Mutat Res. 2005;576:22-38.

122. Li DM and Sun H. PTEN/MMAC1/TEP1 suppresses the tumorigenicity and induces G1 cell cycle arrest in human glioblastoma cells. Proc Natl Acad Sci USA. 1998;95:15406-15411.

123. Li J, Yen C, Liaw D, et al. PTEN, a putative protein tyrosine phosphatase gene mutated in human brain, breast, and prostate cancer. Science. $1997 ; 275: 1943-1947$.

124. Hickman ES, Moroni MC and Helin K. The role of p53 and pRB in apoptosis and cancer. Curr Opin Genet Dev. 2002;12:60-66.

125. Bourdon JC. p53 and its isoforms in cancer. Br J Cancer. 2007;97:277-282. 
126. Vousden KH and Lane DP. p53 in health and disease. Nat Rev Mol Cell Biol. 2007;8:275-283.

127. Platzer P, Upender MB, Wilson K, et al. Silence of chromosomal amplifications in colon cancer. Cancer Res 2002;62:1134-1138.

128. Heldin CH, Miyazono K, ten Dijke P. TGF-beta signalling from cell membrane to nucleus through SMAD proteins. Nature. 1997;390:465-471.

129. Miyaki M and Kuroki T. Role of Smad4 (DPC4) inactivation in human cancer. Biochem Biophys Res Commun. 2003;306:799-804.

130. Tian F, DaCosta Byfield S, Parks WT, et al. Reduction in Smad2/3 signaling enhances tumorigenesis but suppresses metastasis of breast cancer cell lines. Cancer Res. 2003;63:8284-8292.

131. Bale AE and Yu KP. The hedgehog pathway and basal cell carcinomas. Hum Mol Genet. 2001;10:757-762.

132. Romashkova JA, Makarov SS. NF-kappaB is a target of AKT in anti-apoptotic PDGF signalling. Nature. 1999;401:86-90.

133. Shimizu A, O'Brien KP, Sjoblom $\mathrm{T}$, et al. The dermatofibrosarcoma protuberans-associated collagen type Ialpha1/platelet-derived growth factor (PDGF) B-chain fusion gene generates a transforming protein that is processed to functional PDGF-BB. Cancer Res. 1999;59:3719-3723. 


\section{FIGURE LEGENDS:}

Figure 4-1. Summary of recurrent chromosomal aberrations in melanoma. Figure generated with the use of Circos (43).

Figure 4-2. Summary of recurrent chromosomal aberrations in basal cell carcinoma. Figure generated with the use of Circos (43).

Figure 4-3. Summary of recurrent chromosomal aberrations in squamous cell carcinoma. Figure generated with the use of Circos (43).

\section{KEYWORDS:}

aberration; actinic keratosis (AK); amplification; array-CGH; basal cell carcinoma (BCC); chromosome; cutaneous B-cell lymphoma (CBCL); comparative genomic hybridization (CGH); copy number variation (CNV); cutaneous T-cell lymphoma (CTCL); cytogenetic analysis; deletion; dermatofibrosarcoma protuberans (DFSP); fluorescence in situ hybridization (FISH); inversion; isochromosome; karyotype; keratoacanthoma (KA); loss of heterozygosity (LOH); melanoma; merkel cell carcinoma (MCC); metastasis; non-melanoma skin cancer (NMSC); ring chromosome; single nucleotide polymorphism (SNP)-based CNV arrays; skin cancer; skin tumor; squamous cell carcinoma (SCC); squamous cell carcinoma in situ; translocation. 